\title{
Vitamin B12 deficiency mimicking neuroimaging features of motor neuron disease
}

\author{
Deficiência de vitamina B12 mimetizando aspectos de neuroimagem da doença \\ do neurônio motor \\ Wladimir Bocca Vieira de Rezende Pinto', Paulo Victor Sgobbi de Souzaํ, Ricardo Mendes Rogério², \\ José Luiz Pedroso', Orlando Graziani Povoas Barsottini'
}

A 45-year-old man presented with 2-month-history of progressive gait disturbances and behavioral changes. Examination showed vibration sense compromise and pyramidal signs of release. Blood test revealed low serum vitamin B12 (120 pg/mL; normal range $>200 \mathrm{pg} / \mathrm{mL})$. Brain MRI disclosed hyperintense signal in corticospinal tracts, a similar pattern observed in motor neuron disease (MND) (Figure).
Replacement therapy was started and there were motor and cognitive improvements.

Vitamin B12 deficiency may present with different neurological syndromes ${ }^{1}$. Encephalopathy related to B12 deficiency presents with nonspecific white matter changes ${ }^{2}$. Although MTC brain MRI sequence was normal, our patient presented neuroimaging features observed in $\mathrm{MND}^{3}$.
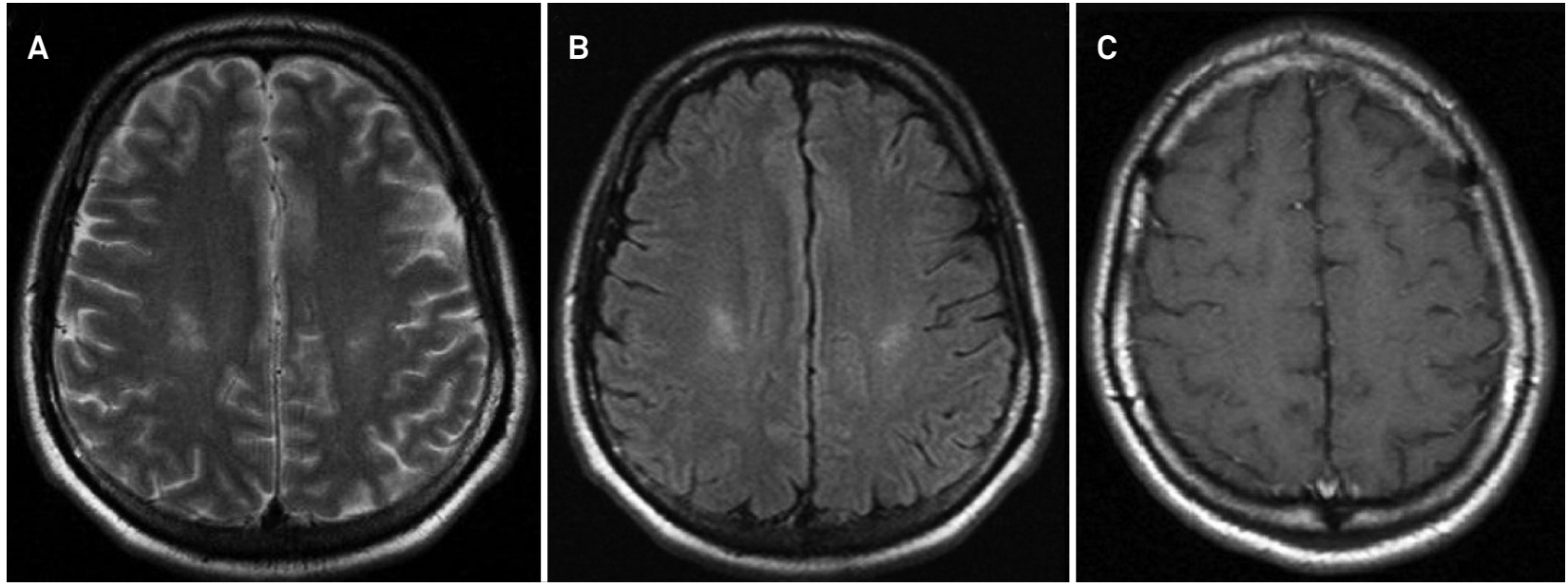

Figure. (A) Axial T2-weighted and (B) axial FLAIR-weighted brain MRI disclosing multiple frontal hyperintense signal changes in corticospinal tracts projections, a similar pattern observed in motor neuron disease. (C) Axial MTC sequence is normal, and no contrast enhancement was observed.

\section{References}

1. Kalita J, Misra UK. Vitamin B12 deficiency neurological syndromes: correlation of clinical, MRI and cognitive evoked potential. J Neurol 2008;255:353-359.
2. Sudo K, Tashiro K. Cerebral white matter lesions associated with vitamin B12 deficiency. Neurology 1998;51:325-326.

3. Lee YC, Markus R, Hughes A. MRI in ALS: corticospinal tract hyperintensity. Neurology 2003;61:1600.

'Departamento de Neurologia, Universidade Federal de São Paulo, Sao Paulo SP, Brazil;

²Departamento de Neurorradiologia, Hospital Beneficência Portuguesa, Sao Paulo SP, Brazil.

Correspondence: Wladimir Bocca Vieira de Rezende Pinto; Rua Botucatu 740; 04023-900 São Paulo SP - Brasil; E-mail:wladimirbvrpinto@gmail.com Conflict of interest: There is no conflict of interest to declare.

Received 15 July 2013; Accepted 23 July 2013. 